\title{
Lu-Hf em zircão por LA-MC-ICP-MS: aplicação em gabro do Ofiolito Aburrá, Colômbia
}

\author{
Anelise Losangela BERTOTTI ${ }^{1}$, Farid CHEMALE JR. ${ }^{2} \&$ Koji KAWASHITA ${ }^{2}$
}

1. Programa de Pós-graduação em Geociências, Instituto de Geociências, Universidade Federal do Rio Grande do Sul. Caixa Postal 15001, 91501-970, Porto Alegre, RS, Brasil. E-mail: anelise.bertotti@ufrgs.br.

2. Instituto de Geociências, Universidade de Brasília. Campus Darcy Ribeiro, Asa Norte, 70910-900, Brasília, DF, Brasil. E-mail: fchemale@ unb.br, koji@usp.br.

Recebido em 09/2011. Aceito para publicação em 06/2013.

Versão online publicada em xx/xx/20xx (www.pesquisasemgeociencias.ufrgs.br)

\begin{abstract}
Resumo - A metodologia Lu-Hf em zircão por LA-MC-ICP-MS implantada no Laboratório de Geologia Isotópica da Universidade Federal do Rio Grande do Sul e aplicada em zircões ígneos de um corpo gabróico do Ofiolito Aburrá, Colômbia, é detalhadamente descrita e constitui o objetivo deste trabalho. O interesse na aplicação desta metodologia, quando se dispõe de um sistema de análise de zircões in situ por LA-MC-ICP-MS, deve ser consignado à relativa simplicidade, sensibilidade e rapidez de análise. A alta concentração de Hf em zircão, que pode alcançar milhares de ppm, propicia razões isotópicas e idades modelos, que possibilitam subsidiar importantes inferências não só quanto à idade de extração mantélica, mas também quanto à sua possível história evolutiva, proveniência e estudos afins. A precisão da ordem de 20 ppm no erro do padrão nas razões ${ }^{176} \mathrm{Hf} /{ }^{177} \mathrm{Hf}$ e de cerca de $10 \%$ nas razões ${ }^{176} \mathrm{Lu} /{ }^{177} \mathrm{Hf}$ propiciada pelo Neptune (ThermoFinnigan) deve ser consignada à moderna tecnologia de medidas simultâneas dos isótopos envolvidos na metodologia. 0 laser de Nd:YAG $(213 \mathrm{~nm})$ da New Wave utilizado revelou-se muito apropriado ao usar os seguintes parâmetros: densidade de energia entre $5 \mathrm{a} 6 \mathrm{~J} / \mathrm{cm}^{2}$, frequência de $10 \mathrm{~Hz}, 50 \mathrm{~s}$ de ablação e diâmetro de $55 \mu \mathrm{m}$. Um fluxo de aproximadamente $4 \mathrm{~mL} / \mathrm{min}$ de nitrogênio adicionado ao gás de arraste para inibir a formação de óxidos junto ao plasma mostrou-se eficaz na estabilização do sinal, resultando em um notável aumento na eficiência de ionização estimado até em 100 \% nos sinais, verificado nos padrões GJ-1 e Mud Tank. Estes padrões são de composições isotópicas ligeiramente diferentes para a razão ${ }^{176} \mathrm{Hf} /{ }^{177} \mathrm{Hf}$ e forneceram valores de $0,282004 \pm$ 0,000004 e $0,282466 \pm 0,000022$, respectivamente. 0 procedimento analítico adotado seguiu um protocolo em que três zircões foram intercalados com o padrão internacional $\mathrm{GJ}$-1. Análises in situ de $\mathrm{Hf}$ em 12 grãos separados do corpo gabróico, previamente datados por $\mathrm{U}-\mathrm{Pb}$, revelaram $\varepsilon_{\mathrm{Hf}}(\mathrm{t})$ entre $+2,01 \mathrm{e}+5,35$, enquanto as idades modelo $\mathrm{T}_{\mathrm{DM}}$ resultaram valores em um intervalo entre 1,15 e 1,44 Ga.
\end{abstract}

Palavras-Chave: metodologia Lu-Hf, zircão, LA-ICP-MS, gabro do Ofiolito Aburrá.

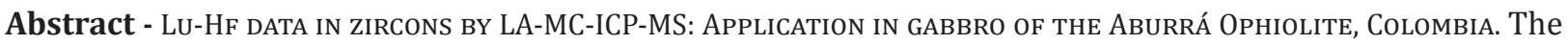
Lu-Hf methodology in zircon by LA-MC-ICP-MS established at the Laboratory of Isotope Geology, Rio Grande do Sul Federal University, and applied in igneous zircons from a gabbroic body of the Aburrá Ophiolite, Colombia, is described. The interest in the application of this methodology when it is available a system of $i n$ situ zircon analysis by LA-ICP-MS, should be consigned to relative simplicity, sensitivity and speed of analysis. The high concentration of $\mathrm{Hf}$ in zircon, which can reach thousands of $\mathrm{ppm}$, provides highly accurate isotopic ratios and model ages that can support not only important inferences about the age of mantle extraction, but also as to its possible evolutionary history, and provenance related studies. The accuracy in order of $20 \mathrm{ppm}$ in standard error of the ${ }^{176} \mathrm{Hf} /{ }^{177} \mathrm{Hf}$ ratios and about $10 \%$ in ${ }^{176} \mathrm{Lu} /{ }^{177} \mathrm{Hf}$ ratios provided by the Neptune (Thermo Finnigan) should be consigned to the modern technology of simultaneous measurements of the isotopes involved in the methodology. The Nd: YAG (213 nm) laser from New Wave used proved to be very appropriate using the following parameters: energy density of $5-6 \mathrm{~J} / \mathrm{cm}^{2}$, frequency of $10 \mathrm{~Hz}, 50 \mathrm{~s}$ of ablation and diameter of $55 \mu \mathrm{m}$. A flow of approximately $4 \mathrm{~mL} / \mathrm{min}$ of nitrogen added to the carrier gas to inhibit the oxide formation in plasma proved to be effective in stabilizing the sign and brought a remarkable increase in ionization efficiency estimated at up to $100 \%$ in the signs, seen in GJ-1 and Mud Tank standards. These standards are slightly different in isotopic compositions for the ${ }^{176} \mathrm{Hf} /{ }^{177} \mathrm{Hf}$ ratio and provided values of $0.282004 \pm 0.000004$ and 0.282466 \pm 0.000022 , respectively. The analytical procedure adopted followed a protocol in which three zircons were interspersed with the international standard GJ-1. In situ analyses of $\mathrm{Hf}$ in 12 grains separated from the gabbroic body, previously dated by $\mathrm{U}-\mathrm{Pb}$, revealed $\varepsilon_{\mathrm{HF}}(\mathrm{t})$ between +2.01 and +5.35 , while the $\mathrm{T}_{\mathrm{DM}}$ model age values resulted in a range between 1.15 and $1.44 \mathrm{Ga}$.

Keywords: Lu-Hf methodology, zircon, LA-MC-ICP-MS, gabbro of Aburrá Ophiolite. 


\section{Introdução}

Durante a última década a eficiência de ionização dos espectrômetros de massa de fonte de plasma indutivamente acoplado contribuiu para que o MC-ICP-MS (Multi Collector - Ion Coupled Plasma - Mass Spectrometer) se tornasse o instrumento mais escolhido para proceder com as análises de Lutécio-Háfnio (Lu-Hf) em zircão. Embora a análise por ablação a laser apresente menor precisão comparada às análises via solução no ICP-MS e ao TIMS, esta desvantagem pode ser superada pela rapidez e praticidade em combinar medidas isotópicas in situ de Hf e U-Pb em um mesmo grão de zircão, de modo a fornecer informações em alta resolução. 0 diâmetro do furo de laser para Hf pode ser até da ordem de $60 \mu \mathrm{m}$, o que não exige muita preocupação com a qualidade do alvo visado ou presença de $\mathrm{Pb}$ comum, como ocorre na obtenção de dados U-Pb. Em termos analíticos, a intensidade do sinal com ICP pode ser até 100 vezes mais intensa do que a obtida com SHRIMP, permitindo sua eficiência na ionização de elementos refratários.

Desta forma, o sistema isotópico Lu-Hf tornou-se uma das ferramentas mais inovadoras e significativas na geocronologia envolvendo zircões. Assim, vem sendo vastamente utilizado como um traçador para entender a evolução crustal e a diferenciação do manto da Terra (Patchett \& Tatsumoto, 1980; Thirwall \& Walder, 1995; Blichert-Toft \& Albarède, 1997; Vervoort \& Blichert-Toft, 1999; Griffinet al., 2000; Woodhead et al., 2004; Gerdes \& Zeh, 2006; Goodge \& Vervoort, 2006; Wu et al., 2006; Nebel et al., 2007; Zeh et al., 2007).

Adicionalmente, o zircão possui alta concentração de $\mathrm{Hf}$ e preserva as razões isotópicas iniciais de Hf, características que favorecem a datação e a indicação petrogenética em estudos de proveniência de sedimentos. A época de derivação mantélica é revelada pela idade modelo conhecida como $\mathrm{T}_{\mathrm{DM}^{\prime}}$, com igual valor interpretativo propiciado originalmente pelo sistema $\mathrm{Sm}-\mathrm{Nd}$. Da mesma forma que a indicação petrogenética é obtida pelo parâmetro $\varepsilon_{\mathrm{Hf}}$ que, quando igualmente comparado com a curva de evolução de Hf para o reservatório condrítico (CHUR), fornece a assinatura isotópica do possível ambiente de formação (manto ou crosta) do magma.

Assim, o estudo combinado envolvendo o método $\mathrm{U}-\mathrm{Pb}$, um dos métodos geocronológicos mais significativos, à assinatura isotópica propiciada pelo $\mathrm{Hf}$ e idade modelo $\mathrm{T}_{\mathrm{DM}}$ de Lu-Hf permite inferir o magma dos quais os zircões derivaram e cristalizaram. Em uma formação geológica as diferentes assinaturas isotópicas de Hf encontradas em uma população de zircão ou mesmo em um único zircão permitem caracterizar diferentes eventos magmáticos ou metamórficos que aconteceram ao nível regional durante a evolução crustal das regiões de origem (Gerdes \& Zeh, 2006; Gerdes \& Zeh, 2009).

Neste trabalho será descrito, de forma detalhada, a metodologia Lu-Hf para LA-MC-ICP-MS em zircão, desenvolvida no Laboratório de Geologia Isotópica da Universidade Federal do Rio Grande do Sul. Nesse contexto, ainda serão apresentadas análises isotópicas de Hf obtidas por dois padrões de zircão, GJ-1e Mud Tank, e seus resultados com adição de nitrogênio durante as análises. Por fim, será realizada a aplicação desta metodologia nos zircões do Gabro do Ofiolito Aburrá, Colômbia, previamente datados pelo método U-Pb.

\section{Sistema isotópico Lu-Hf}

Os primeiros estudos isotópicos envolvendo $\mathrm{Hf}$ foram feitos por Patchett \& Tatsumoto (1980) utilizando o clássico procedimento para análises isotópicas de elementos sólidos conhecido abreviadamente por TIMS (Thermal Ionization Mass Spectrometer). A separação de Lu e Hf em colunas de troca catiônica (Patchett et al., 1981) foi necessária para evitar interferências isobáricas e melhorar a precisão nas medidas das razões isotópicas do Hf. No entanto, com a necessidade da ordem de 1 micrograma de Hf para obtenção de resultados precisos em razão da baixa eficiência de ionização térmica (Blichert-Toft \& Albarède, 1997) as análises isotópicas passaram gradualmente a ser executadas com ICP-MS, pois alguns mL da solução de Hf com algumas dezenas de ppb são suficientes.

$\mathrm{O}$ elemento $\mathrm{Hf}$ é muito abundante, alcançando $10.000 \mathrm{ppm}$ em muitos zircões pelo fato do mesmo substituir facilmente o $\mathrm{Zr}$ na sua estrutura (Hoskin \& Schaltegger, 2003). 0 Hf pertence ao grupo IVB (valência +4), constitui elemento de elevado potencial iônico (HFSE - High Field Strength Element), possui raio iônico de $0,81 \AA$ A e propriedades químicas similares ao do zircônio $\left(\mathrm{Zr}^{+4}, 0,80 \AA \AA\right)$. Além do zircão, o Hf pode ser também encontrado em alta concentração na badeleíta, em torno de 1,33\% (Faure, 2005).

O Lu é o último elemento na faixa dos Terras Raras Pesados (ETRP), trivalente, insere-se no grupo dos lantanídeos e é o elemento que apresenta o menor raio atômico neste grupo $(0,93 \AA ̊)$. O Lu está presente em todos os tipos de rocha (Faure, 1986), mas em concentrações baixas e sempre associado ao itérbio (Yb). 0 mineral que apresenta maior concentração de Lu é a badeleíta, cerca de $70 \mathrm{ppm}$. 0 Lu também é encontrado em outros minerais como zircão, granada, monazita e o xenotímio (Kinny \& Maas, 2003; Faure, 2005).

O Hf possui seis isótopos naturais: ${ }^{174} \mathrm{Hf}(0,16 \%)$, ${ }^{176} \mathrm{Hf}(5,2 \%),{ }^{177} \mathrm{Hf}(18,6 \%),{ }^{178} \mathrm{Hf}(27,1 \%),{ }^{179} \mathrm{Hf}(13,63$ $\%)$ e ${ }^{180} \mathrm{Hf}(35,1 \%)$. Já o Lutécio possui somente dois isótopos, o ${ }^{175} \mathrm{Lu}(97,4 \%)$ e o ${ }^{176} \mathrm{Lu}(2,59 \%)$. Sendo que o isótopo ${ }^{176} \mathrm{Lu}$ é um radionuclídeo que decai espontaneamente para o ${ }^{176} \mathrm{Hf}$ liberando uma partícula beta. Devido a este decaimento, o ${ }^{176} \mathrm{Hf}$ aumenta com o tempo nas rochas e nos minerais e a idade Lu-Hf de rochas e minerais pode ser calculada a partir da equação 1: 
$\frac{{ }^{176} H f}{{ }^{177} H f}=\left(\frac{{ }^{176} H f}{{ }^{177} H f}\right)_{I}+\frac{{ }^{176} L u}{{ }^{177} H f}\left(e^{\lambda t}-1\right)$

Neste cálculo o t é o tempo decorrido desde a formação da rocha ou do mineral e o $\lambda$ é a constante de decaimento de ${ }^{176} \mathrm{Lu}$. Atualmente, ainda se tem incerteza referente ao valor adequado a ser assumido para a constante de decaimento. Baseado em meteoritos (eucritos) Patchett \& Tatsumoto (1980) propuseram o valor de $1,94 \times 10^{-11} \mathrm{a}^{-1}$. 0 trabalho mais recente de Söderlund et al. (2004) sugere um valor mais preciso e um pouco menor $(3,8 \%)$ de $1,867 \times 10^{-11} \mathrm{a}^{-1}$. Para o presente estudo foi assumido o valor de $1,867 \times 10^{-11} \mathrm{a}^{-1}$ proposto por Söderlund e colaboradores que se basearam em resultados de Lu-Hf de diferentes minerais de doleritos Sorkka e Karlshemn da Suécia e Finlândia.

O zircão hospeda isótopos de vida longa tais como U e Th cujas concentrações podem atingir 1\% ou até mais, além dos produtos de decaimento e ETR's cujas concentrações são variáveis, porém na faixa de ppm. A concentração de Lu é baixa, sendo a razão Lu/Hf normalmente menor que 0,002 . Assim, a razão ${ }^{176} \mathrm{Hf} /{ }^{177} \mathrm{Hf}$ do zircão pode ser considerada como sendo o valor inicial de quando ele foi cristalizado. Outra característica que torna o zircão um mineral de interesse é sua alta resistência para eventos termais posteriores, pois a temperatura de fechamento do Hf no zircão é de $200^{\circ} \mathrm{C}$, de modo a demonstrar que o $\mathrm{Hf}$ permanece isotopicamente fechado durante a maioria dos eventos termais (Faure, 1986). 0 zircão é um mineral acessório comum em inúmeras rochas e preserva uma complexa história de crescimento. Portanto, núcleos herdados e crescimentos metamórficos no zircão podem ser usados para identificar e distinguir vários processos geológicos.

O Sm e o Nd são elementos geoquimicamente muito parecidos. Os raios iônicos são semelhantes, da mesma forma que carga e eletronegatividade. Já o Lu e o Hf são diferentes em carga e raio. Apesar destas diferenças, as propriedades geoquímicas de Lu-Hf são similares ao par Sm-Nd ao tratarem de seus comportamentos geoquímicos na fusão parcial do manto, pois $\mathrm{Hf}$ em relação ao Lu é mais concentrado no líquido silicático assim como o Nd para o Sm. Desta forma, os magmas basálticos derivados do manto têm razões Lu/Hf mais baixas que a da rocha fonte, assim como o sólido residual é empobrecido em Hf e tem razão Lu/Hf mais alta que a rocha antes da diferenciação. Adicionalmente a presença ou não da granada no manto possibilita tal diferenciação, pois a granada desempenha um papel importante por ter afinidade com Lu e inibir sua entrada no material fundido (Faure, 2005).

Devido a estas características, a metodologia Lu-Hf possibilita, além da determinação da idade de diferenciação, estabelecer um indicador petrogenético denominado de $\varepsilon_{\mathrm{Hf}}$ O parâmetro $\varepsilon_{\mathrm{Hf}}$ consiste basicamente na comparação da razão ${ }^{176} \mathrm{Hf} /{ }^{177} \mathrm{Hf}$ da amostra estudada para a época de sua formação ou de seu valor atual, relacionado ao reservatório condrítico uniforme padrão (CHUR). Este fator auxilia na identificação das fontes de magmas e de processos de formação de rochas e mineralizações e é expresso conforme a equação 2:

$$
\varepsilon_{H f}=\left[\frac{\left({ }^{176} \mathrm{Hf} /{ }^{177} \mathrm{Hf}\right)_{a m}^{j}}{\left({ }^{176} \mathrm{Hf} /{ }^{177} \mathrm{Hf}\right)_{\mathrm{CHUR}}^{t}}-1\right] \times 10^{4}
$$

Partindo do pressuposto que na época de cristalização da rocha seu magma progenitor tiver uma razão ${ }^{176} \mathrm{Hf} /{ }^{177} \mathrm{Hf}$ mais elevada que o condrito, então o $\varepsilon_{\mathrm{Hf}}$ será positivo. Isso significa que a fonte deste magma possuía uma razão Lu/Hf mais elevada que a do condrito, ou seja, a fonte é o manto. Por outro lado, caso na formação da rocha, seu magma progenitor possuísse uma razão ${ }^{176} \mathrm{Hf} /{ }^{177} \mathrm{Hf}$ menor que a do condrito, o valor de $\varepsilon_{\mathrm{Hf}}$ seria negativo e, portanto, a fonte destas rochas teria uma razão Lu/Hf menor que a do condrito, como é o caso dos magmas de origem crustal. Portanto, quando o parâmetro $\varepsilon_{H f}$ apresenta valores positivos a fonte em geral é de origem mantélica, e quanto maior for este valor positivo mais empobrecido geoquimicamente em Hf é este manto do qual o material se derivou.

Outra informação que pode ser obtida pela metodologia é a idade modelo do "manto empobrecido" (DM - Depleted Mantle). Tal modelo admite que o manto sofrera episódios de fracionamento envolvendo a extração de magmas basálticos para a geração de crosta continental e causou o empobrecimento em elementos leves (LILE - Large Ion Lithophile Elements) incluindo elementos terras raras leves (ETRL) neste reservatório (Nebel et al., 2007). 0 resultado dessas diferenciações é um manto residual enriquecido na razão Lu/Hf e empobrecido geoquimicamente em Hf. A idade modelo do manto empobrecido pode ser calculada pela equação 3 :

$$
T_{D M}=\frac{t_{(U / P b)}}{1000}+\frac{1}{0.01867} \times \ln \left(1+\frac{\left({ }^{176} \mathrm{Hf} /{ }^{177} \mathrm{Hf}\right)_{a m}^{t}-\left({ }^{176} \mathrm{Hf} /{ }^{177} \mathrm{Hf}\right)_{D M}^{t}}{\left({ }^{176} \mathrm{Lu} /{ }^{177} \mathrm{Hf}\right)_{\text {crosta }}-\left({ }^{176} \mathrm{Lu} /{ }^{177} \mathrm{Hf}\right)_{D M}^{0}}\right)
$$

Assim, os valores atuais assumidos para o manto empobrecido para as razões ${ }^{176} \mathrm{Hf} /{ }^{177} \mathrm{Hf}$ e ${ }^{176} \mathrm{Lu} /{ }^{177} \mathrm{Hf}$ são iguais a 0,28325 e 0,0388 , respectivamente (Andersen et al., 2009). A idade $\mathrm{T}_{\mathrm{DM}}$ é calculada a partir da composição isotópica inicial de Hf do momento em que o zircão cristalizou e da razão Lu/Hf da crosta precur- sora, seja ela de composição máfica ou félsica (Pietranik et al., 2008). Portanto, é importante que as análises de U-Pb e Lu-Hf sejam efetuadas sobre a mesma porção de um grão de zircão, a fim de recalcular o $\varepsilon_{\mathrm{Hf}}$ e a idade $\mathrm{T}_{\mathrm{DM}}$ do momento de sua cristalização. 


\section{Procedimentos analíticos}

\subsection{MC-ICP-MS}

As análises de Hf em zircão foram realizadas utilizando o MC-ICP-MS Neptune (ThermoFinnigan) um espectrômetro de massa multicoletor de alta resolução para medidas de razões isotópicas com uma con- figuração especial para detectar simultaneamente uma ampla gama de nuclídeos de elementos como ${ }^{202} \mathrm{Hg}$ até ${ }^{238} \mathrm{U}$. O Neptune é equipado com nove coletores Faraday e seis contadores de íons, que podem ser combinados em diversas configurações. A configuração dos coletores utilizada para as análises de Lu-Hf acha-se ilustrada na tabela 1 e todas as análises foram realizadas no modo estático e em baixa resolução.

Tabela 1. Configuração dos coletores Faraday adotada para as análises de Lu e Hf.

\begin{tabular}{c|cccccccc}
\hline & L4 & L3 & L2 & L1 & C & H1 & H2 & H3 \\
\hline Isótopos & ${ }^{171} \mathrm{Yb}$ & ${ }^{173} \mathrm{Yb}$ & ${ }^{174} \mathrm{Hf}$ & ${ }^{175} \mathrm{Lu}$ & ${ }^{176} \mathrm{Hf}$ & ${ }^{177} \mathrm{Hf}$ & ${ }^{178} \mathrm{Hf}$ & ${ }^{179} \mathrm{Hf}$ \\
Interferentes & & & & ${ }^{174} \mathrm{Yb}$ & & ${ }^{176}(\mathrm{Yb}+\mathrm{Lu})$ & & \\
\hline
\end{tabular}

\subsection{Laser}

A microssonda a laser (Laser Abalation - LA), modelo UP213 (Nd:YAG) da New Wave, utilizada para as análises por ablação em minerais pode emitir alta densidade de energia em vários tamanhos de crateras (4 $\mu \mathrm{m}-110 \mu \mathrm{m})$. 0 gás hélio $(\mathrm{He})$ é o responsável pelo transporte do material da ablação para o ICP com um fluxo de 0,35-0,45 L/min. Para as análises de Hf uma pequena quantidade de nitrogênio $\left(\mathrm{N}_{2}\right)$ da ordem de 3,5 a $4 \mathrm{~mL} / \mathrm{min}$ foi adicionada ao gás Ar de arraste, conforme Louie \& Soo(1992) que aplicaram esta mistura, para diminuir a formação de óxidos no plasma e aumentar a intensidade do sinal em mais de $100 \%$ em algumas análises, conforme detalhado mais adiante.

Durante as análises de Hf com laser foi utilizada uma taxa de repetição de $10 \mathrm{~Hz}$. Dependendo do tipo de laser utilizado e parâmetros selecionados constituem- -se de uma prática corrente valer-se de uma frequência de 10 a 20 pulsos por segundo. Com o laser UP213 de $\mathrm{Nd}$ :YAG, a taxa definida acima revelou-se satisfatória em termos de eficiência e estabilidade dos sinais. Diâmetro dos furos com laser foram de $55 \mu \mathrm{m}$ fornecendo um sinal intenso da ordem de 3 volts para ${ }^{178} \mathrm{Hf}$ em todas as análises de Hf. De acordo com as quantidades de Hf dos padrões GJ-1 e Mud Tank, uma potência de $60 \%$ do laser mostrou-se adequada, de modo a fornecer uma densidade de energia de $5-6 \mathrm{~J} / \mathrm{cm}^{2}$. A sequência de análises empregada intercalou três grãos da amostra com um dos padrões, preferencialmente $G J-1$ em que a razão ${ }^{176} \mathrm{Hf} /{ }^{177} \mathrm{Hf}$ é conhecida com precisão. Os grãos foram montados em uma pastilha de epoxi com uma pequena cavidade para inserção do cilindro do padrão. Todos os parâmetros de instrumentação e operação usados para as análises de Lu-Hf em zircão por LA-ICP-MS estão resumidos na tabela 2.

Tabela 2. Parâmetros de instrumentação e operação para o Laser Ablation e o MC-ICP-MS.

\begin{tabular}{ll}
\hline MC-ICPMS & \\
\hline $\begin{array}{l}\text { Instrumento } \\
\text { Fonte de íon } \\
\text { Potência }\end{array}$ & $1200 \mathrm{~W}$ \\
Fluxo de gás & Ar Resfriador: $15 \mathrm{~L} / \mathrm{min}$ \\
& Ar Auxiliar: $0,7 \mathrm{~L} / \mathrm{min}$ \\
& Ar Transporte: $0,72-0,76 \mathrm{~L} / \mathrm{min}$ \\
& $\mathrm{N}_{2}$ Transporte: $3,5-4,5 \mathrm{~mL} / \mathrm{min}$ \\
& $-2000 \mathrm{~V}$ \\
Extração & Estático \\
Modo de Análise & Coletores Faraday \\
Detecção & \\
Aquisição de Dados & $1,049 \mathrm{~s}$ \\
Tempo de integração & 1 \\
No de integrações & \\
\hline Laser Ablation & $\mathrm{UP}-213$ (Nd:YAg) New Wave \\
Instrumento & $55 \mu \mathrm{m}$ \\
Diâmetro do furo & $5-6 \mathrm{~J} / \mathrm{cm}^{2}$ \\
Energia & $10 \mathrm{~Hz}$ \\
Frequência & $50 \mathrm{~s}$ \\
Total da ablação & $0,32 \mathrm{a} 0,45 \mathrm{~L} / \mathrm{min}$ \\
Fluxo de He &
\end{tabular}




\subsection{Cálculos da correção de interferência isobárica}

Os isótopos de Lu, Hf e Yb são medidos simultaneamente durante as análises no MC-ICP-MS. As razões isotópicas medidas destes elementos durante os aproximadamente $50 \mathrm{~s}$ de análise foram: ${ }^{173} \mathrm{Yb} /{ }^{171} \mathrm{Yb}$, ${ }^{179} \mathrm{Hf} /{ }^{177} \mathrm{Hf},{ }^{175} \mathrm{Lu} /{ }^{177} \mathrm{Hf},{ }^{176} \mathrm{Hf} /{ }^{177} \mathrm{Hf}$. Tendo em vista a necessidade de correção do fracionamento isotópico que ocorre durante as análises, as razões isotópicas de $\mathrm{Yb}$ foram normalizadas assumindo para ${ }^{173} \mathrm{Yb} /{ }^{171} \mathrm{Yb} \mathrm{o}$ valor de 1,1301 (Segal et al., 2003), da mesma forma as razões isotópicas de $\mathrm{Hf}$ e Lu foram normalizadas assumindo o valor de 0,7325 para a razão ${ }^{179} \mathrm{Hf} /{ }^{177} \mathrm{Hf}$ (Patchett \& Tatsumoto, 1980). Assim, com base no objetivo de testar as correções do Yb foram preparadas soluções do padrão JMC-475 (200 ppb) dopado com quantidades conhecidas de $\mathrm{Yb}(\mathrm{Yb} / \mathrm{Hf}=0,05$ e 0,1$)$ fornecendo o valor de 0,282149 $\pm 0,000031$ ( $n=6,2 D P$ ), em concordância com o valor aceito de 0,282159 \pm 0,000038 (Chu et al., 2002).

Durante as análises in situ de Hf no zircão, os nuclídeos isóbaros interferentes, ${ }^{176} \mathrm{Lu}$ e ${ }^{176} \mathrm{Yb}$, são medidos juntamente com o ${ }^{176} \mathrm{Hf}$. Para se obter a razão ${ }^{176} \mathrm{Hf} /{ }^{177} \mathrm{Hf}$ do zircão com acurácia, as interferências devidas a ${ }^{176} \mathrm{Lu} \mathrm{e}{ }^{176} \mathrm{Yb}$ foram cuidadosamente corrigidas com base nos sinais observados do ${ }^{175} \mathrm{Lu}$ e do ${ }^{173} \mathrm{Yb}$, ambos com as devidas normalizações. A intensidade do sinal correspondente ao ${ }^{176} \mathrm{Hf}$ foi calculada através da equação 4:

$$
{ }^{176} H f={ }^{176}(H f+L u+Y b)_{m}-\left[\begin{array}{l}
{ }^{175} L u_{m} \times\left(\frac{{ }^{176} L u}{{ }^{175} L u}\right)_{N} \times\left(\frac{M_{176(L u)}}{M_{175}}\right)^{\beta(L u)} \\
+{ }^{173} Y b_{m} \times\left(\frac{{ }^{176} Y b}{{ }^{173} Y b}\right)_{N} \times\left(\frac{M_{176(Y b)}}{M_{173}}\right)^{\beta(Y b)}
\end{array}\right]
$$

Os fatores $\beta_{(\mathrm{Lu})}$ e o $\beta_{(\mathrm{Yb})}$ são os fatores de fracionamento para Lu e $\mathrm{Yb}$. Estes dois fatores variam durante o tempo da análise no grão de zircão (Iizuka \& Hirata, $2005)$, isto indica que o fator $\beta$ não pode ser assumido como uma constante durante as análises, devendo ser calculado para cada medida realizada.

Por fim, os dados são processados em planilhas Excel e específicas para Lu-Hf a fim de calcular a idade modelo $\mathrm{T}_{\mathrm{DM}}$ e o parâmetro $\varepsilon_{\mathrm{Hf}}$ do ponto analisado. Nestas planilhas são selecionados aproximadamente 45 dados de modo a descartar as primeiras medidas de um total de 50 dados integrados de 1,049 s/ponto.

\section{Resultados}

\subsection{Análises dos padrões de $\mathrm{Hf}$}

Em termos de estabilidade do sinal, as análises de solução com MC-ICP-MS apresentam razões isotópicas mais precisas do que por ablação a laser. Desta forma, antes de iniciar as sessões de ablação a laser, o espectrômetro é rotineiramente focalizado e aferido com o padrão em solução posterior a calibração de ganho dos amplificadores Faraday. Em um total de 24 análises da solução pura do padrão de Hf JMC-475 (200 ppb) a razão média ${ }^{176} \mathrm{Hf} /{ }^{177} \mathrm{Hf}$ normalizada foi de $0,282156 \pm$ $0,000015(\mathrm{n}=24,2 \mathrm{DP})$.

No decorrer das análises in situ a laser, dois padrões foram utilizados para verificar a reprodutibilidade do aparelho. Um dos padrões conhecido por $G J-1$ (Elhlou et al., 2006; Morel et al., 2008) e fornecido pela GEMOC (Geochemical Evolution and Metallogeny of
Continents, MacQuarie University, Sydney, Australia), foi extraído de um pegmatito africano com idade de cristalização de 608,5 \pm 0,4 Ma. As cores dos grãos deste padrão variam do vermelho ao amarelado e as imagens de CL (catodo luminescência) mostram significativa homogeneidade. 0 outro padrão é o zircão conhecido como Mud Tank (Woodhead \& Hergt, 2005), fornecido por Elena Belousova. Trata-se de um padrão internacional para Lu/Hf separado de um carbonatito aflorante no Strangways Range situado a noroeste da Austrália e cuja idade U-Pb foi estabelecida como sendo de 732 Ma. Zircões e apatitas são encontrados em abundância e os tamanhos podem ser maiores que $10 \mathrm{~cm}$.

$\mathrm{O}$ valor obtido para a razão ${ }^{176} \mathrm{Hf} /{ }^{177} \mathrm{Hf}$ do $G J-1$ durante as análises dos zircões do gabro do Ofiolito Aburrá (ver seção 4.2) foi de 0,282004 $\pm 0,000004$ ( $n=3,2 \mathrm{DP})$, em perfeita concordância com o valor de $0,282000 \pm 0,000004$ publicado (Morel et al., 2008). A respectiva razão para o Mud Tank foi de 0,282466 \pm $0,000022(n=3,2 D P)$ bastante concordante com a razão $0,282504 \pm 0,000044$ publicada por Woodhead \& Hergt (2005).

Valores destes padrões também foram obtidos após diversos testes com diâmetros de 40 e $55 \mu \mathrm{m}$ e com nitrogênio. Os resultados são apresentados na tabela 3. Em geral, as análises com $55 \mu \mathrm{m}$ e nitrogênio mostraram intensidades altas no ${ }^{178} \mathrm{Hf}$ com valores em torno de 2,6 a 3,2 V no GJ-1 e de 3,13 a 3,27 V no Mud Tank, mostrando um aumento de mais de $100 \%$ se compararmos com as demais análises que obtiveram intensidades em torno de 0,89 e 2,13 V. As medidas da razão ${ }^{176} \mathrm{Hf} /{ }^{177} \mathrm{Hf}$ também foram mais precisas com 55 $\mu \mathrm{m}$ e nitrogênio do que as demais análises com um va- 
lor de 0,282010 $\pm 0,000012(\mathrm{n}=3,2 \mathrm{DP})$ para o $G J-1$ e 0,282472 $\pm 0,000028(\mathrm{n}=3,2 \mathrm{DP})$ para o Mud Tank. A razão ${ }^{176} \mathrm{Lu} /{ }^{177} \mathrm{Hf}$ apresentou erros baixos em todas as análises e manteve boa reprodutibilidade no GJ-1, enquanto no Mud Tank não foi obtido a mesma reprodutibilidade devido ao baixo teor de ${ }^{176} \mathrm{Lu}$ deste padrão.

Na figura 1 são apresentados os resultados das análises de Hf no padrão GJ-1 com $55 \mu \mathrm{m}$, com e sem adição de nitrogênio. Nas análises é possível verificar que as intensidades de Hf no decorrer das mesmas decrescem razoavelmente na medida em que o furo vai ficando com maior profundidade. 0 índice de fracionamento calculado para as razões isotópicas de Hf é próximo a 1, o que indica um leve fracionamento durante a ablação.

Tabela 3. Resultados dos padrões GJ-1 e Mud Tank durante as análises e testes utilizando 40 e 55 um e nitrogênio.

\begin{tabular}{c|cccccc}
\hline Padrão & ${ }^{{ }^{178}} \mathbf{H f}_{\text {max }}$ (V) & ${ }^{176} \mathbf{H f} /{ }^{177} \mathbf{H f}$ & 2 DP & ${ }^{176} \mathbf{L u} /{ }^{177} \mathbf{H f}$ & 2 DP & Observações \\
\hline $\begin{array}{c}\text { Análises } \\
\text { GJ-1 }\end{array}$ & 2,34 & 0,282004 & 0,000004 & 0,000259 & 0,000008 & $\mathrm{n}=3,55 \mu \mathrm{m}, \mathrm{N}_{2}$ \\
Testes & & & & & & \\
GJ-1 & 2,91 & 0,282010 & 0,000012 & 0,000283 & 0,000012 & $\mathrm{n}=3,55 \mu \mathrm{m}, \mathrm{N}_{2}$ \\
GJ-1 & 1,72 & 0,282067 & 0,000022 & 0,000275 & 0,000012 & $\mathrm{n}=3,55 \mu \mathrm{m}$ \\
GJ-1 & 1,40 & 0,282044 & 0,000062 & 0,000261 & 0,000012 & $\mathrm{n}=3,40 \mu \mathrm{m}, \mathrm{N}_{2}$ \\
GJ-1 & 0,89 & 0,282040 & 0,000026 & 0,000258 & 0,000024 & $\mathrm{n}=3,40 \mu \mathrm{m}$ \\
\hline Análises & & & & & & \\
Mud Tank & 3,19 & 0,282466 & 0,000022 & 0,000104 & 0,000012 & $\mathrm{n}=3,55 \mu \mathrm{m}, \mathrm{N}_{2}$ \\
Testes & & & & & & \\
Mud Tank & 3,20 & 0,282472 & 0,000028 & 0,000069 & 0,000020 & $\mathrm{n}=3,55 \mu \mathrm{m}, \mathrm{N}_{2}$ \\
$\begin{array}{c}\text { Mud Tank } \\
\text { Mud Tank }\end{array}$ & 2,13 & 0,282563 & 0,000046 & 0,000046 & 0,000004 & $\mathrm{n}=3,55 \mu \mathrm{m}$ \\
Mud Tank & 1,54 & 0,282455 & 0,000032 & 0,000074 & 0,000004 & $\mathrm{n}=3,40 \mu \mathrm{m}, \mathrm{N}_{2}$ \\
\hline
\end{tabular}

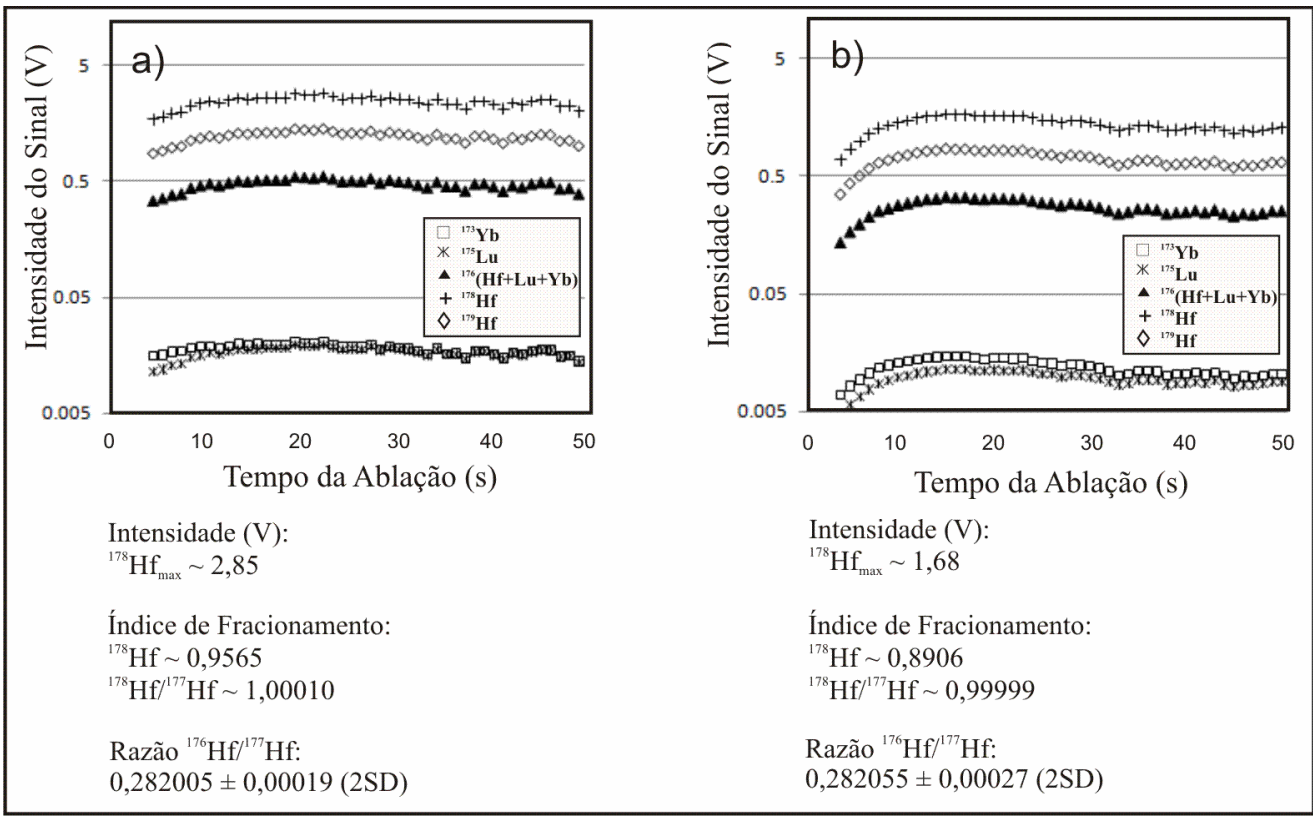

Figura 1. Exemplo de comparação dos dados isotópicos de Hf, intensidade do sinal e índice de fracionamento no padrão GJ-1; A) 55 m e com nitrogênio e B) 55 m e sem nitrogênio.

\subsection{Aplicação da metodologia Lu-Hf}

Para este estudo usou-se zircões separados de um gabro pegmatítico pertencente ao Ofiolito Aburrá, localizado no setor norte da Colômbia, Cordilheira Central (Fig. 2). 0 ofiolito consiste em três unidades: o Dunito de Medellín composto por rochas ultramáficas, os me- tagabros de El Picacho com rochas plutônicas máficas e os metasedimentos de médio a alto grau (Correa et al., 2005). 0 mesmo se encontra em contato tectônico sobre rochas metamórficas mais antigas do que o Triássico Médio e é intrudido por rochas graníticas do Jurássico e do Cretáceo (Martínez, 2007).

Martínez (2007) realizou estudos petrográficos, 
geoquímicos e isotópicos nos corpos ultramáficos e gabróicos da unidade em questão, os quais apresentam características petrográficas e químicas similares as rochas de mesma composição do Ofiolito de Omã. A idade U-Pb em zircão obtida de um plagiogranito foi de $216 \pm 0,4 \mathrm{Ma}$ e os dados de isótopos de Sm-Nd sugerem que as rochas gabróicas tenham sido derivadas do manto empobrecido. Com base nestes estudos, Martinez (2007) pôde concluir que o conjunto de rochas máfico-ultramáficos da região representa um ofiolito, denominando-os de Ofiolito Aburrá. Neste contexto, os dados de Lu-Hf nos zircões do gabro deste ofiolito contribuem para o estudo da geologia da borda NW da América do Sul.

Os zircões do Ofiolito Aburrá foram primeiramente datados pelo método U/Pb por LA-MC-ICP-MS e forneceram uma idade concordante de $228 \pm 0,92 \mathrm{Ma}$ (Restrepo et al., 2007), obtida a partir de seis zircões concordantes (Fig. 3). A preparação da amostra, assim como a montagem da sessão com os zircões, seguiu a sistemática descrita em Chemale Jr. et al. (2012). Os zircões separados foram montados sobre um cilindro epóxi e as imagens foram feitas com o microscópio eletrônico de varredura (MEV). Os grãos medem em torno de $500 \mu \mathrm{m}$, de modo geral mostram-se bem formados e homogêneos (Fig. 4). A idade U-Pb obtida para os zircões do ofiolito é a idade que foi utilizada para o cálculo da idade modelo $\mathrm{T}_{\mathrm{DM}}$ de $\mathrm{Hf}$ conforme equação 3 e também das razões ${ }^{176} \mathrm{Hf} /{ }^{177} \mathrm{Hf}$ iniciais. Tal idade de $228 \mathrm{Ma}$ é interpretada como sendo a idade de cristalização do magma gabróico hospedeiro, Triássico Superior.

As análises in situ de $\mathrm{Hf}$ foram efetuadas em doze zircões da mesma sessão utilizada para datação por $\mathrm{U}-\mathrm{Pb}$, seguindo a metodologia descrita na seção 3.2 deste trabalho. Os furos para as análises com laser foram feitos próximos dos alvos selecionados para as análises U-Pb (Fig. 4) para evitar eventuais dúvidas interpretativas. Na tabela 4 são apresentados os valores para o $\varepsilon_{\mathrm{Hf}}(\mathrm{t})$ obtidos entre $+2,01 \mathrm{a}+5,35$ e das idades modelos que se enquadraram entre 1,15 a $1,44 \mathrm{Ga}$. Na figura 5 são ilustrados através de diagramas os valores de $\varepsilon_{\mathrm{Hf}}(\mathrm{t})$ e da razão inicial ${ }^{176} \mathrm{Hf} /{ }^{177} \mathrm{Hf}$ obtidos para a idade de cristalização de 228 Ma destes zircões.

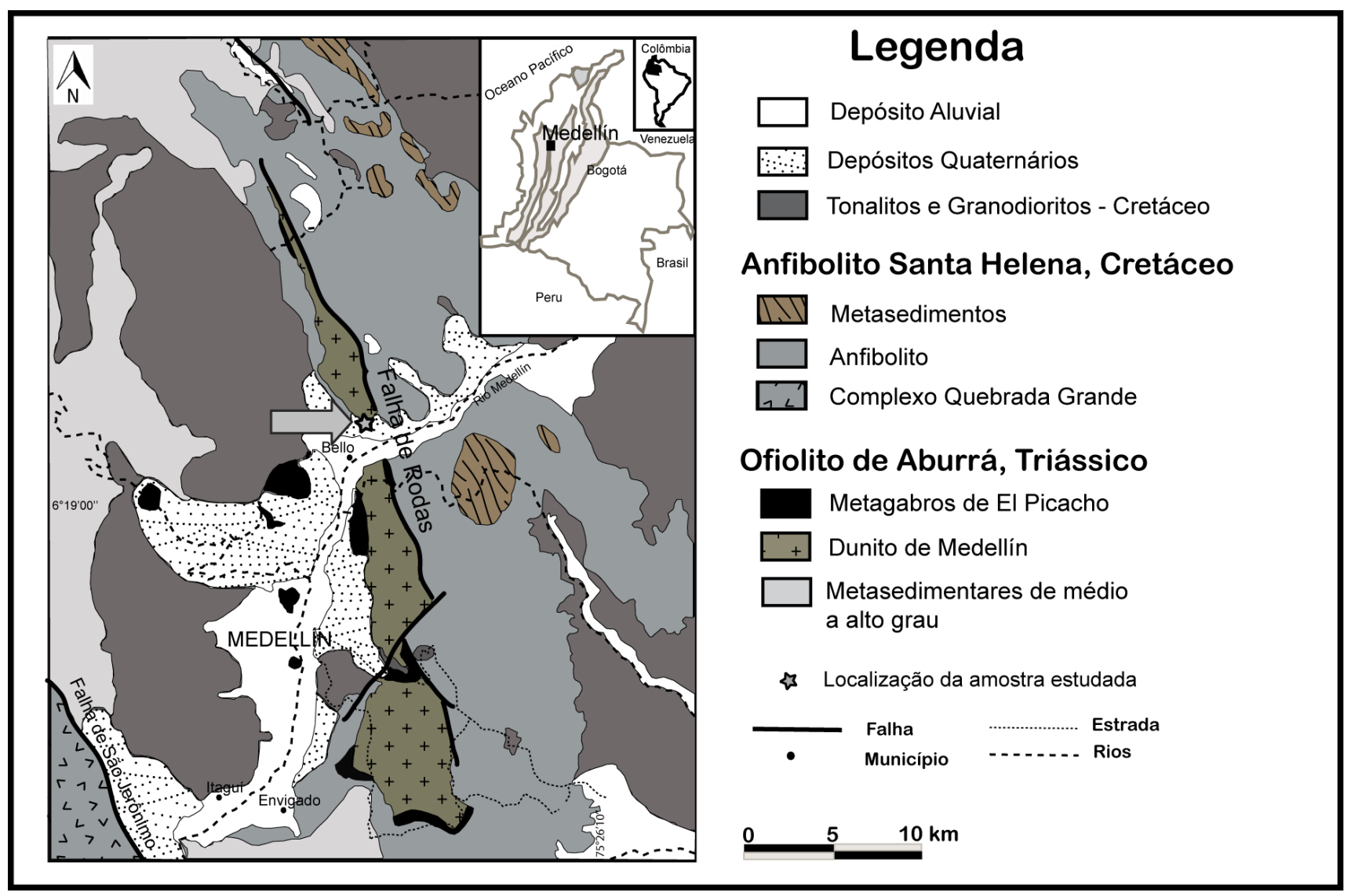

Figura 2. Mapa geológico da cordilheira central da Colômbia mostrando a geologia da região de Medellín e localização da amostra estudada (modificado de Restrepo et al., 2012). 


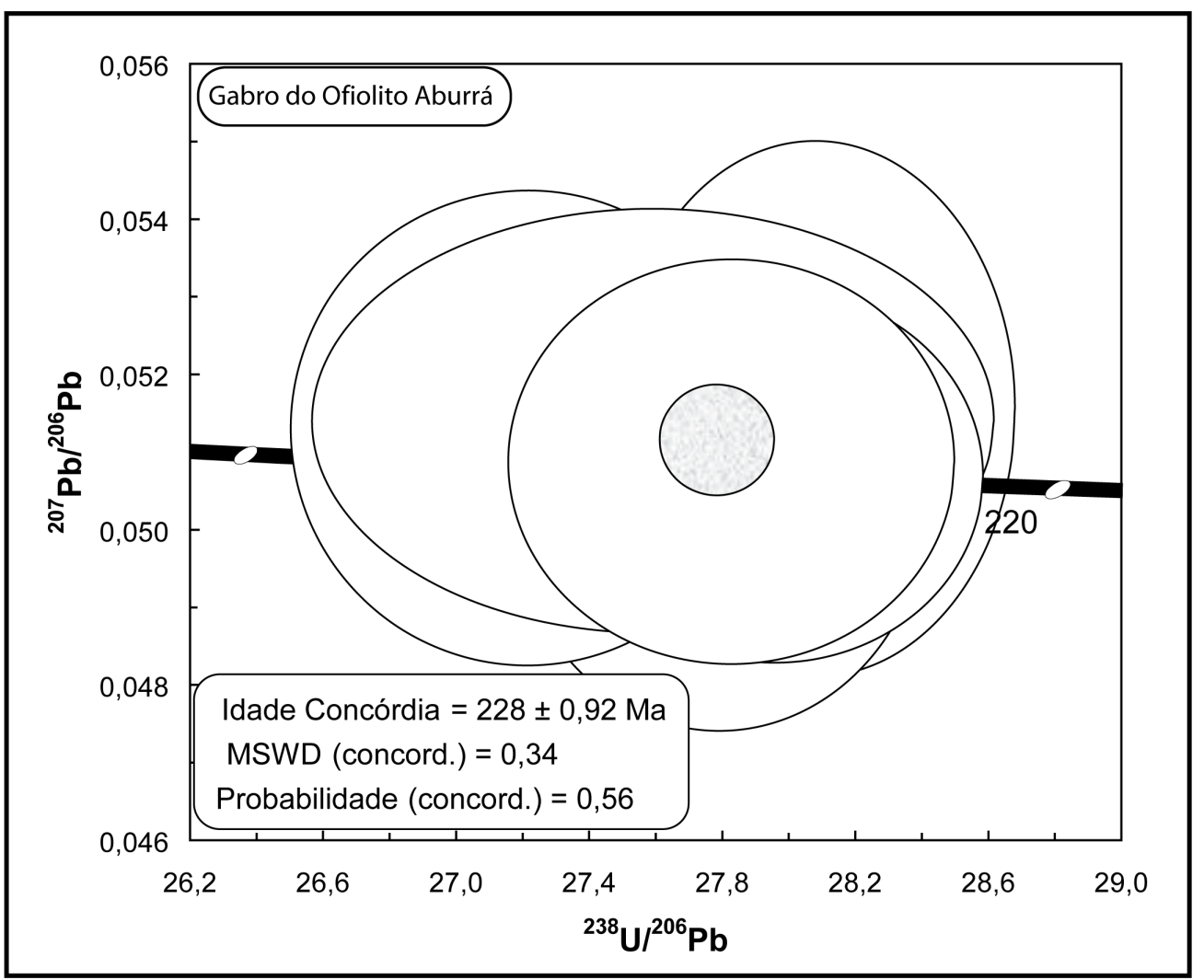

Figura 3. Diagrama concórdia com dados de U/Pb em zircão da amostra de gabro pertencente ao Ofiolito Aburrá obtidos com o LA-MC-ICP-MS (Restrepo et al., 2007).
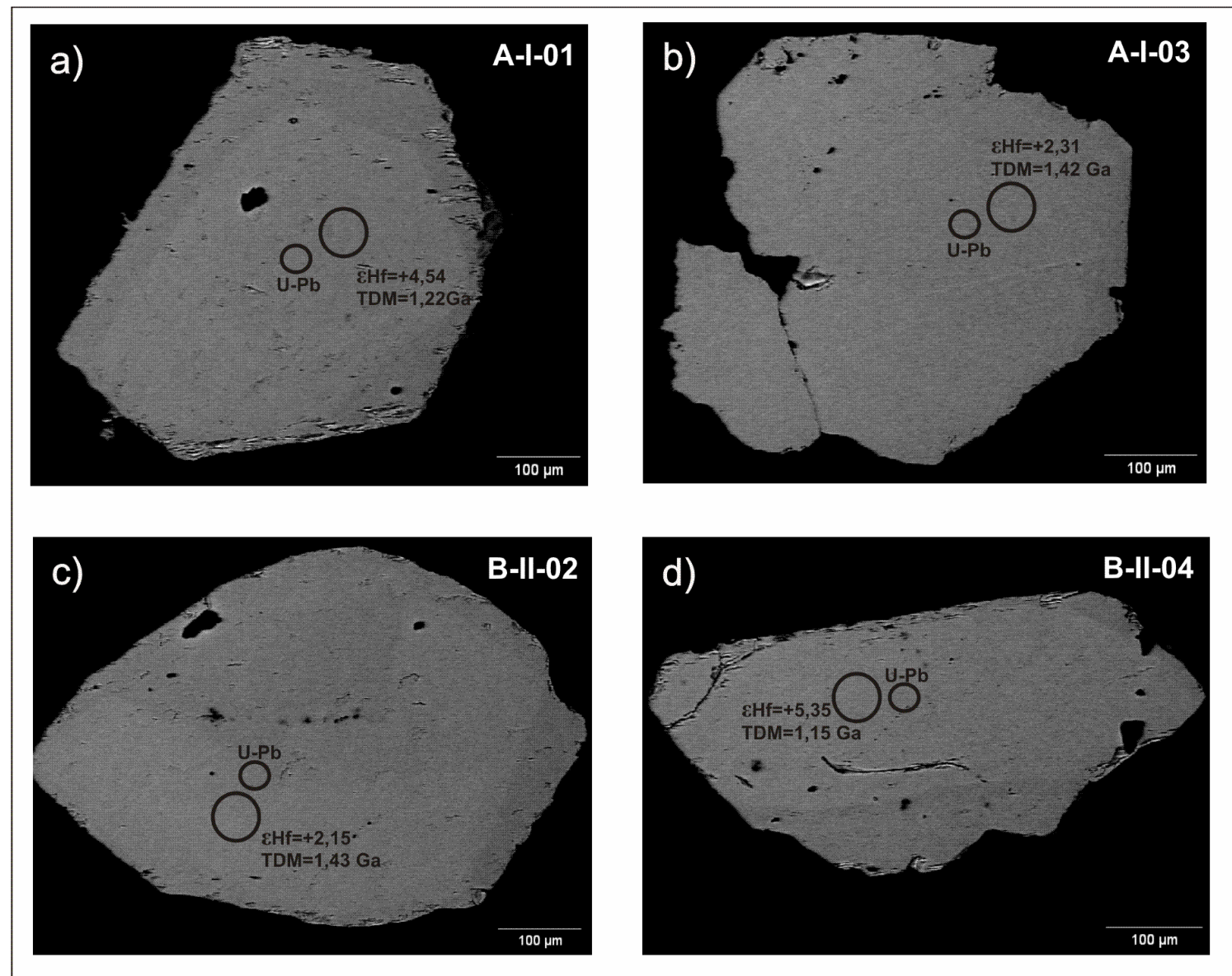

Figura 4. Imagem de MEV de alguns zircões do Gabro do OfiolitoAburrá ilustrando os furos feitos nas análises de U-Pb (furo menor) e Lu-Hf. a) Zircão A-I-01, b) Zircão A-I-03, c) Zircão B-II-02, d) Zircão B-II-04. 
Tabela 4. Resultados das análises de Hf nos zircões do gabro pertencente ao Ofiolito Aburrá.

\begin{tabular}{|c|c|c|c|c|c|c|c|}
\hline Nome & ${ }^{176} \mathrm{Hf} /{ }^{177} \mathrm{Hf}$ & $\pm 2 \mathrm{SE}$ & ${ }^{176} \mathrm{Lu} /{ }^{177} \mathrm{Hf}$ & $\pm 2 \mathrm{SE}$ & ${ }^{176} \mathrm{Hf} /{ }^{177} \mathrm{Hf}(\mathrm{t})$ & $\varepsilon_{\mathrm{Hf}}(\mathrm{t})$ & $\begin{array}{c}\text { Idade } \\
\mathbf{T}_{\mathrm{DM}}\end{array}$ \\
\hline Zr-065-A-I-01 & 0,282773 & 0,000033 & 0,000606 & 0,000008 & 0,282770 & 4,54 & 1,22 \\
\hline Zr-065-A-I-03 & 0,282708 & 0,000031 & 0,000219 & 0,000007 & 0,282707 & 2,31 & 1,42 \\
\hline Zr-065-A-I-06 & 0,282718 & 0,000033 & 0,000620 & 0,000011 & 0,282715 & 2,60 & 1,39 \\
\hline Zr-065-B-II-01 & 0,282700 & 0,000024 & 0,000325 & 0,000003 & $0,2 \varepsilon$ & 2,01 & 1,44 \\
\hline Zr-065-B-II-02 & 0,282705 & 0,000029 & 0,000594 & 0,000011 & 02 & 2,15 & 1,43 \\
\hline Zr-065-B-II-03 & 0,2 & $0,00 c$ & 0,00 & 0,00 & 65 & 4,37 & 1,24 \\
\hline Zr-0 & 6 & 2 & 4 & 0,0 & 93 & 5,35 & 1,15 \\
\hline Zr-0 & 34 & 00029 & 72 & 0 & 83 & 5,00 & 1,18 \\
\hline Zr-06 & 30 & & & & 27 & 3,02 & 1,36 \\
\hline Zr-065-B-II-07 & 0,282734 & & & & & 3,26 & 1,33 \\
\hline Zr-06 & 0,282738 & 0,000032 & & & 0,282734 & 3,28 & 1,33 \\
\hline Zr-065-C-III-01 & 0,282716 & 0,000018 & 0,000595 & 0,000002 & 0,282713 & 2,53 & 1,40 \\
\hline
\end{tabular}

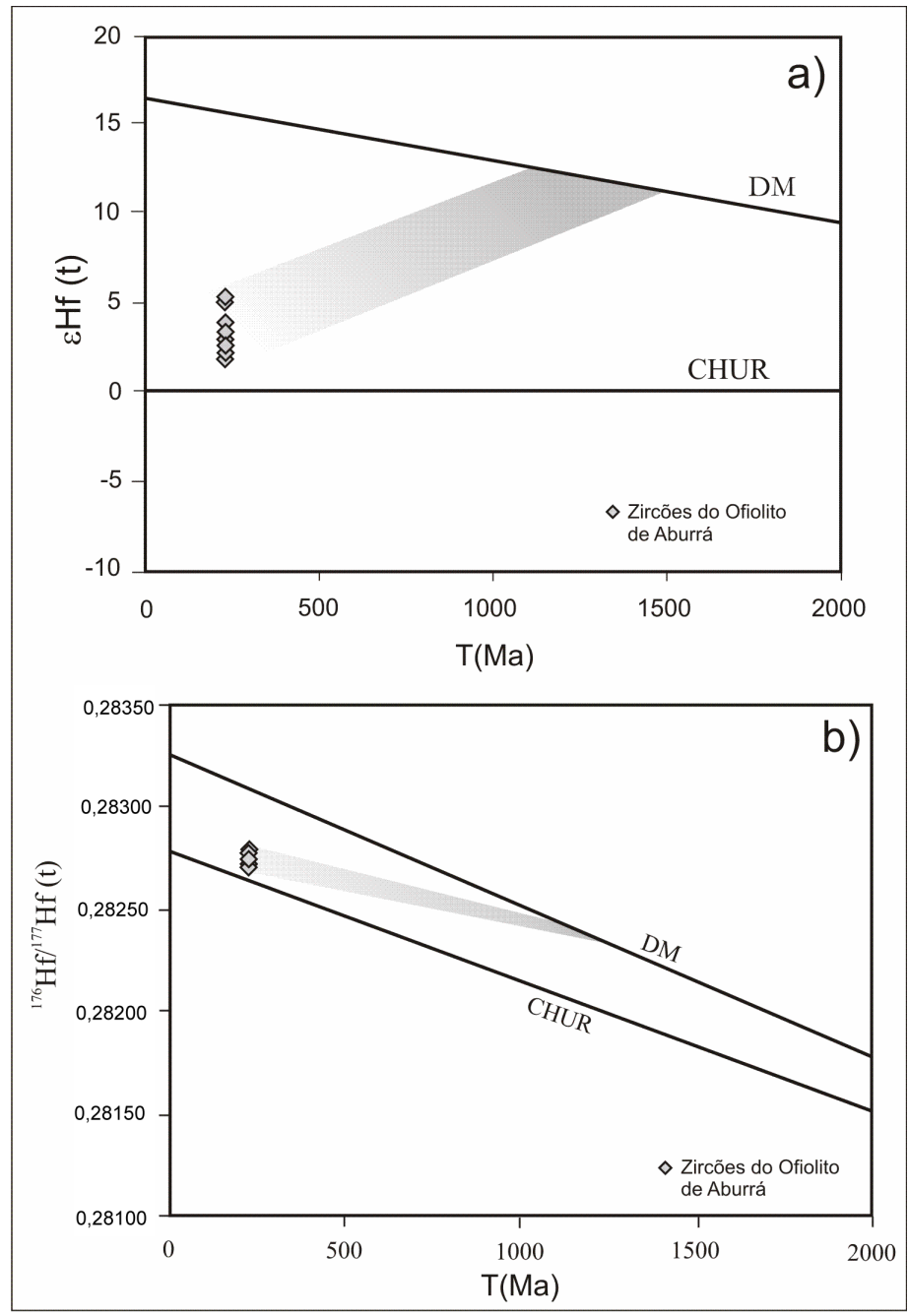

Figura 5. Diagramas dos valores de $\varepsilon H f(t)$ (A) e da evolução isotópica de Hf (B) dos zircões do Gabro do OfiolitoAburrá. As áreas sombreadas indicam a tendência de evolução para o gabro. 


\section{Discussão dos resultados}

Na trajetória das análises de Hf o nitrogênio foi injetado por meio de um fluxômetro e conexão $\mathrm{T}$ ao gás de arraste (Ar) com a finalidade de aumentar a sensibilidade do sinal na obtenção das razões isotópicas do Hf. Nos testes efetuados com padrões foi comprovado que o uso deste gás a uma vazão de aproximadamente $4 \mathrm{~mL} / \mathrm{min}$ aumentava significativamente a intensidade dos sinais. Também foi observada a diferença de sinal entre um furo com $55 \mu \mathrm{m}$ de diâmetro contra um de $40 \mu \mathrm{m}$. A diferença a favor de 55 mícrons é notória, de tal forma que, quando possível, é preferível usar diâmetros maiores. Na literatura constam diâmetros até da ordem de 100 mícrons.

A idade U/Pb de 229 Ma é a idade de cristalização do magma gabróico hospedeiro. Os valores de $\varepsilon_{\mathrm{Hf}}(\mathrm{t}) \mathrm{ob}$ tidos nesta unidades são positivos, o que sugere a presença de um magma juvenil com uma possível contaminação crustal, o que explicaria a variabilidade destes valores se aproximando de zero. A despeito das razões $\mathrm{Lu} / \mathrm{Hf}$ serem determinadas com baixa precisão devido ao baixo teor de Lu (cerca de11 ppm, por exemplo, no padrão GJ-1) não afeta de forma significativa as idades modelos calculadas.

Assim, as idades $\mathrm{T}_{\mathrm{DM}}$ obtidas de 1,15-1,44 Ga podem ser interpretadas como a idade de formação do zircão ou que o manto que gerou o magma gabróico tenha sido um manto modificado. Esta faixa da idade $\left(\mathrm{T}_{\mathrm{DM}}\right)$ de Hf coincide bem com os eventos magmáticos do mesoproterozoico na Plataforma Sul-Americana, com boa expressão no sistema Sunsás-Aguapeí (1,2-1,1 Ga) e contemporânea à formação das rochas da Província Rondoniana - San Ignacio (1,5-1,3 Ga) (e.g.: Tohver et al., 2004; Santos et al., 2008).

Os dados de Hf aqui apresentados são semelhantes aos dados já publicados de zircões desta área por Matteini et al. (2008). As diferenças maiores são verificadas nas idades modelo $\mathrm{T}_{\mathrm{DM}}$. Aqueles autores obtiveram valores entre $0,6-0,7 \mathrm{Ga}$, cerca de $50 \%$ mais baixos que os obtidos neste trabalho. A explicação para esta diferença é justificada pela utilização, naquele caso, do valor médio crustal igual a 0,0113 na razão ${ }^{176} \mathrm{Lu} /{ }^{177} \mathrm{Hf}$ no cálculo da idade $\mathrm{T}_{\mathrm{DM}}$ (ver equação 3 ). No presente trabalho utilizou-se um valor de 0,022 para esta mesma razão, que se refere a uma crosta precursora de composição máfica (Pietranik et al., 2008), sendo coerente com a área de estudo.

\section{Conclusões}

A metodologia Lu-Hf descrita e aplicada no Laboratório de Geologia Isotópica da UFRGS para análises in situ de zircões por LA-MC-ICP-MS mostrou-se confiável conforme esperado. Os dois padrões GJ-1 e Mud Tank utilizados durante as análises reproduziram os valores da literatura na margem de erro usuais. Verificou-se que o uso de nitrogênio durante as análises de Hf comprovou ser efetivo, não só em aumento na sensibilidade, mas também na estabilidade dos sinais, propiciando um ganho significativo na precisão dos resultados obtidos com análises in situ do sistema Lu-Hf em zircões.

A metodologia Lu-Hf aplicada aos zircões do Gabro do Ofiolito Aburrá forneceu novos dados isotópicos e idades modelos que aparentemente seriam mais coerentes com as recentes ideias da conexão da Província Amazônica e o Laurentiano contexto de amalgamação do Continente Rodinia ou de geração de um magma a partir de manto modificado. Os dados combinados $\mathrm{U}-\mathrm{Pb}$ e Lu-Hf são ainda muito escassos para maiores interpretações, mas possibilitam antever o potencial instrumento para robustecer melhor as ideias e intensificar a elaboração conceitual acerca da evolução crustal, não só em nível regional, mas também em nível global.

Agradecimentos- Este trabalho foi realizado com apoio do CNPq, Conselho Nacional de Desenvolvimento Científico e Tecnológico através da bolsa de doutorado (ALB). Os autores agradecem a Antonio Celso (Sens Representações Ltda.) pela cessão de um fluxômetro regulável para nitrogênio utilizado pioneiramente no país para as análises do sistema Lu-Hf, a J.J. Restrepo, da Universidade Nacional da Colômbia, e a J.C. Frantz, da UFRGS, pelo fornecimento dos zircões da amostra de gabro.

\section{Referências}

Andersen, T., Andersson, U.B., Graham, S., Åberg, G. \&Simonsen, S.L. 2009. Granitic magmatism by melting of juvenile continental crust: new constraints on the source of Palaeoproterozoic granitoids in Fennoscandia from Hf isotopes in zircon. Journal of Geological Society, 166: 233-247.

Blichert-Toft, J. \&Albarède, F. 1997. The Lu-Hf isotope geochemistry of chondrites and the evolution of the mantle crust system. Earth and Planetary Science Letters,148: 243-258.

Chemale Jr., F., Kawashita, K., Dussin, I.A., Justino, D. \&Bertotti, A.L.2012. U-Pb zircon in situ dating with LA-MC-ICPMS using mixed detector configuration. Anais da Academia Brasileira de Ciências, 84: 275-296.

Chu, N.C., Taylor, R.N., Chavagnac, V., Nesbitt, R.W., Boella, M. \& Milton, J.A. 2002. Hf isotope ratio analysis using multi-collector inductively coupled plasma mass spectrometry: an evaluation of isobaric interference corrections. Journal of Analytical Atomic Spectrometry, 17: 1567-1574.

Correa, A.M., Martens, U.C., Restrepo, J.J., Ordoñez-Carmona, 0. \& Pimentel, M. 2005. Subdivisión de lãs metamorfitas básicas de losalrededores de Medellín, Cordillera Central de Colombia. Colombia. Revista Academia Colombiana de Ciencias, 29 (112): 325-344.

Elhlou, S., Belousova, E., Griffin, W.L., Pearson, N.J. \&O’Reilly, S.Y. 2006. Trace element and isotopic composition of GJred zircon standard by laser ablation. Geochimica et Cosmochimica Acta, 70(18): 158.

Faure, G. 1986. Principles of Isotope Geology. New York, John Wiley \& Sons, $2^{\text {a} E d ., ~ 589 p . ~}$

Faure, G. 2005. Isotopes: Principles and Applications. New York, John Wiley \& Sons, $3^{\mathrm{a}} \mathrm{Ed}$., 897p.

Gerdes, A. \& Zeh, A. 2006. Combined U-Pb and Hf isotope 
LA-(MC)-ICP-MS analyses of detrital zircons: Comparison with SHRIMP and new constraints for the provenance and age of Amorican metasediment in central Germany. Earth and Planetary Science Letters, 249: 47-61.

Gerdes, A. \& Zeh, A. 2009. Zircon formation versus zircon alteration - New Insights from combined $\mathrm{U}-\mathrm{Pb}$ and $\mathrm{Lu}-$ Hf in-situ LA-ICP-MS analyses, and consequences for the interpretation of archean zircon from the Central Zone of the Limpopo Belt. Chemical Geology, 261: 230-243.

Goodge, J.W. \& Vervoort, J.D. 2006. Origin of Mesoproterozoic A-type granites in Laurentia: Hf isotope evidence. Earth and Planet Science Letters, 243:711-731.

Griffin, W.L., Pearson, N.J., Belousova, E., Jackson, S.E., van Achterbergh E., O’Reilly S.Y. \& Shee, S.R. 2000. The Hf isotope composition of cratonic mantle: LAM-MC-ICPMS analysis of zircon megacrysts in Kimberlites. Geochimica et Cosmochimica Acta, 64: 133-147.

Hoskin, P. \&Schaltegger, U. 2003. The Composition of Zircon and Igneous and Metamorphic Petrogenesis. Reviews in Mineralogy and Geochemistry, 53: 27-62.

Iizuka, T. \& Hirata, T. 2005. Improvements of precision and accuracy in situ Hf isotope microanalysis of zircon using the laser ablation-MC-ICPMS technique. Chemical Geology, 220:131-137.

Louie, H. \& Soo,S.Y.-P.1992. Use of nitrogen and hydrogen in inductively plasma mass spectrometry. Journal of Analytical Atomic Spectrometry, 7: 557-564.

Kinny, P. \& Maas, R. 2003. Lu-Hf and Sm-Nd Isotope systems in zircon. Reviews in Mineralogy and Geochemistry, 53: 327-341.

Martínez, A.M.C. 2007. Petrogênese e Evolução do Ofiolito de Aburrá, Cordilheira Central dos Andes Colombianos. Brasília, 204p. Tese de Doutorado, Programa de Pós-graduação em Geologia, Instituto de Geociências, Universidade de Brasília.

Matteini, M., Restrepo, J.J., Correa, A. \& Pimentel, M.M. 2008. Lu-Hf LA-MC-ICP-MS In Situ Analyses on Petrographic Thin Section: Application on Zircon Macrocrysts from Aburrá Ophiolite, Central Cordillera of Colombia. In: SOUTH AMERICAN SYMPOSIUM ON ISOTOPE GEOLOGY, 6, CD-Rom, San Carlos de Bariloche, Argentina.

Morel, M.L.A., Nebel, O., Nebel-Jacobsen, Y.J., Miller, J.S. \& Vroon, P.Z. 2008. Hafnium isotope characterization of the GJ-1 zircon reference material by solution and laser-ablation MC-ICPMS. Chemical Geology, 255: 231-235.

Nebel, O., Nebel-Jacobsen, Y., Mezger, K. \& Berndt, J. 2007. Initial Hf isotope compositions in magmatic zircon from early Proterozoic rocks from the Gawler Craton, Australia: A test for zircon model ages. Chemical Geology, 241: 23-37.

Patchett, P.J. \& Tatsumoto, M. 1980. Lu-Hf total rock isochron for the eucrite meteorites. Nature, 288: 571-574.

Patchett, P.J., Kouvo, O., Hedge, C.E. \& Tatsumoto, M. 1981. Evolution of continental crust and mantle heterogeneity: evidence from Hf isotopes. Contribution to Mineralalogy and Petrology, 78: 279-297.

Pietranik, A.B., Hawkesworth, C.J., Storey, C.D., Kemp, A.I.S., Sircombe, K.N., Whitehouse, M.J. \& Bleeker, W. 2008. Episodic, mafic crust formation from 4.5 to $2.8 \mathrm{Ga}$ : New evidence from detrital zircons, Slave craton, Canada. The
Geological Society of America, 36(11): 875-878.

Restrepo A., J.J., Frantz, J.C., Ordóñez-Carmona, O., Correa, A.M., Martens, U. \& Chemale, F. 2007. Edad triásica de formación de la Ofiolita de Aburrá, flanco occidental de lacordillera Central. In: CONGRESO COLOMBIANO DE GEOLOGÍA,11, Anais,Bucaramanga.

Restrepo A., J.J., Ibañez-Mejía, M. \& Garcia-Casco, A. 2012. $\mathrm{U}-\mathrm{Pb}$ zircon ages of the Medellin amphibolites (Central Cordillera of Colombia) reveal mid-Cretaceous tectonic juxtaposition of Triassic and mid-Cretaceous metamorphic complexes. In: South American Symposium on Isotope Geology, 8, Anais, Medellín.

Santos, J.O.S., Rizzotto, G.J., Potter, P.E., McNaughton, N.J., Matos, R.S., Hartmann, L.A., Chemale Jr., F. \& Quadros, M.E.S. 2008. Age and autochthonous evolution of the Sunsás Orogen in West Amazon Craton based on mapping and UPb geochronology. Precambrian Research, 165: 120-152.

Segal, I., Halicz, L. \& Platzner, I.T. 2003. Accurate isotope ratio measurements of ytterbium by multiple collection inductively coupled plasma mass spectrometry applying erbium and hafnium in an improved double external normalization procedure. Journal of Analytical Atomic Spectrometry, 18: 1217-1223.

Söderlund, U., Patchett, J.P, Vervoort, J.D. \& Isachsen, C.E. 2004. The ${ }^{176} \mathrm{Lu}$ decay constant determined by Lu-Hf and $\mathrm{U}-\mathrm{Pb}$ isotope systematics of Precambrian mafic intrusions. Earth and Planetary Science Letters, 219: 311-324.

Thirwall, M.F. \& Walder, A. J. 1995. In situ hafnium isotope ratio analyses of zircon by inductively coupled plasma mass spectrometry. Chemical Geology, 122: 241-247.

Tohver, E., Bettencourt, J.S., Tosdal, R., Mesger, K., Leite, W.B. \& Payolla, B.L. 2004.Terrane transfer during the Grenville orogeny: tracing the Amazonian ancestry of southern Appalachian basement through $\mathrm{Pb}$ and $\mathrm{Nd}$ isotopes. Earth and Planetary Science Letters, 228: 161-176.

Vervoort, J. \& Blichert-Toft, J. 1999. Evolution of the depleted mantle: Hf isotope evidence from juvenile rocks through time. Geochimica et Cosmochimica Acta, 63: 533-557.

Woodhead, J., Hergt, J., Shelley, M., Eggins, S. \& Kemp, R. 2004. Zircon Hf-isotope analysis with an excimer laser, depthprofiling, ablation of complex geometries and concomitant age stimation. Chemical Geology, 209: 121-135.

Woodhead, J.D. \& Hergt, J. M. 2005. A preliminary appraisal of seven natural zircon reference materials for in situ hf isotope determination. Geostandards and Geoanalytical Research, 29: 183-195.

Wu, F.-Y., Yang, Y.-H., Xie, L.-W., Yang, J.-H. \& Xu, P. 2006. Hf isotopic compositions of the standard zircons and baddeleyites used in U-Pb geochronology. Chemical Geology, 234: 105-126.

Zeh, A., Gerdes, A., Klemd, R. \& Barton Jr., J.M. 2007. Archaean to Proterozoic Crustal Evolution in the Central Zone of the Limpopo Belt (South Africa-Botswana): Constraints from Combined U-Pb and Lu-Hf Isotope Analyses of Zircon. Journal of Petrology, 48: 1605-1639.

Manuscrito 484

Editores: Edinei Koester e Paulo A. Souza 